\title{
Enhancement of Signal Intensity for Inverse Photoelectron Spectroscopy by Surface Plasmon Resonance
}

\author{
Ryota Usui ${ }^{1}$, Hiroyuki Yoshida ${ }^{1,2}$ \\ 1 Graduate School of Engineering, Chiba University, 1-33, Yayoi-cho, Inage-ku, Chiba, \\ 263-8522, Japan \\ 2 Molecular Chirality Research Center, Chiba University, 1-33, Yayoi-cho, Inage-ku, \\ Chiba, 263-8522, Japan
}

Supporting Information

1. Evaluation Methods of Extinction Spectra

2. Extinction Spectra of CuPc/Ag Nanoparticles

3. LEIPS Spectra of CuPc/Ag and Derivation of CuPc Contributions 


\section{Evaluation Method of Extinction Spectra}

The photon incident to the sample is either absorbed by the sample, transmitted, or reflected. Figure $\mathrm{S} 1$ shows transmittance $T$ and reflectance $R$ of the Ag nanoparticles and the $\mathrm{CuPc} / \mathrm{Ag}$ measured using the integrating sphere. We determined extinction ratio as 1

$-T-R$ which is shown in Figure 2c. The obtained extinction spectrum of CuPc/Ag contains both the absorption by SPR of Ag nanoparticle and that of CuPc molecules. The further process to separate the SPR contribution is described in the next section. 

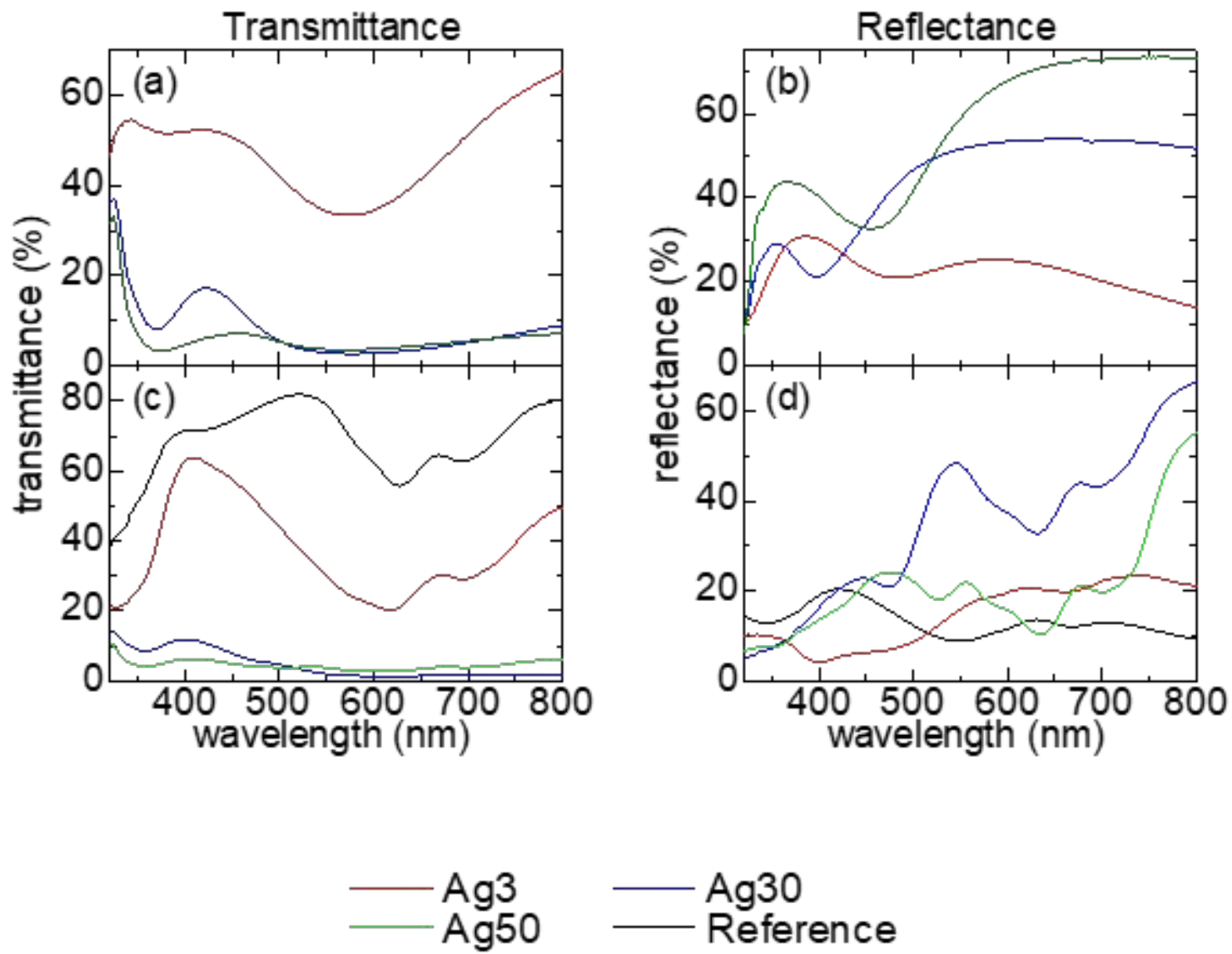

Figure S1. Results of optical measurements. (a) the transmittance and (b) the reflectance spectra of Ag nanoparticles. (c) the transmittance and (d) the reflectance spectra of CuPc/Ag. 


\section{Extinction Spectra of CuPc / Ag Nanoparticles}

Figure S2 shows the extinction spectra obtained using the method described in the previous section. The Q-band of CuPc is clearly visible in the range between 550 and 750 $\mathrm{nm}$. We remove the absorption due to the CuPc molecules from the extinction spectrum of $\mathrm{CuPc} / \mathrm{Ag}$ by subtracting the log of extinction (corresponding to the absorbance) of CuPc /ITO from that of CuPc/Ag extinction spectra. The resultant extinction spectra owing to SPR of Ag nanoparticle are shown in Figure 3c.

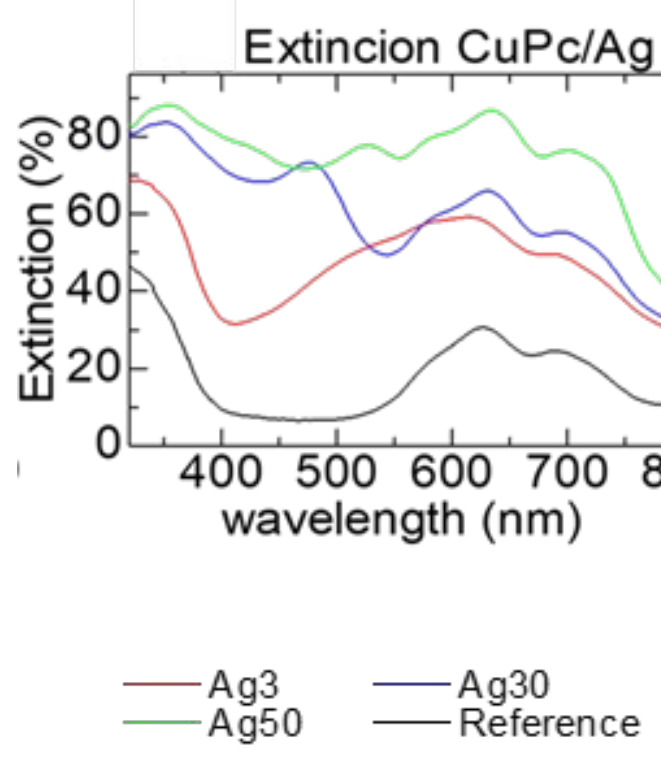

Figure S2. Extinction spectrum obtained by the procedure described in the previous section. 


\section{Raw LEIPS Spectra of CuPc/Ag and Derivation of CuPc}

\section{Contributions}

Figure S3 shows the LEIPS spectrum measured at $335 \mathrm{~nm}$ in wavelength as a function of average CuPc film thickness. The spectra are composed of the Ag- and CuPc-derived components. The Ag-derived spectrum shows a sharp rise at about $0.5 \mathrm{eV}$ owing to the increase of the electron current and is almost constant in intensity. On the other hand, the CuPc-derived spectrum shows a peak around $1.5 \mathrm{eV}$. As the film thickness increases, the CuPc-derived intensity increases and the Ag-derived intensity decreases.

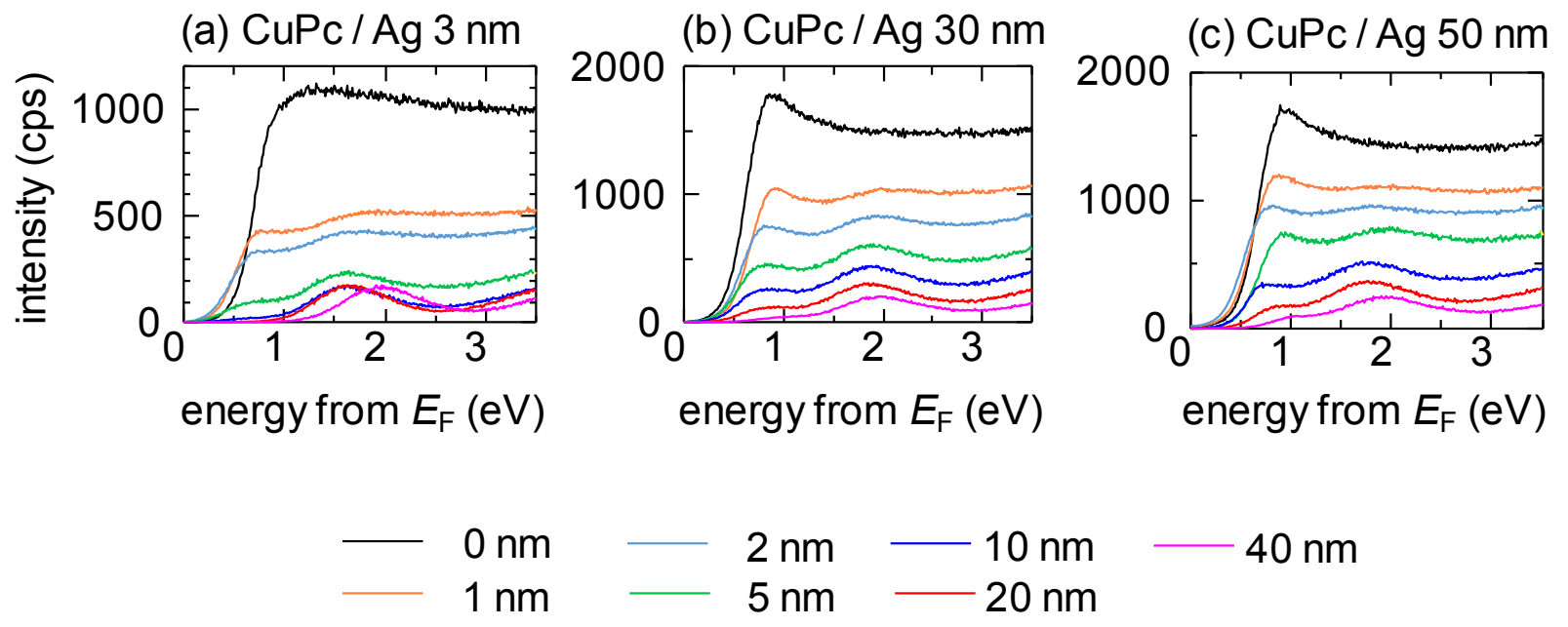

Figure S3. LEIPS spectra of CuPc/Ag films as a function of thickness. (a) CuPc/Ag3 (b) CuPc/Ag30 (c) CuPc/Ag50. The detection wavelength is $335 \mathrm{~nm}$. 
To discuss the enhancement by SPR of CuPc-derived LEIPS signal, we subtracted the Ag-derived LEIPS spectra from the measured LEIPS spectra of CuPc/Ag. Figure S4 shows the procedure for $\mathrm{CuPc}(5 \mathrm{~nm}) / \mathrm{Ag}(3 \mathrm{~nm})$ as an example. The spectrum of pristine Ag nanoparticles is multiplied by 0.15 so as to match the LEIPS spectrum of Ag of CuPc I Ag around the rising edge (Figure S4a). Figure S4b shows the resultant difference spectrum showing the CuPc-derived LEIPS spectra.
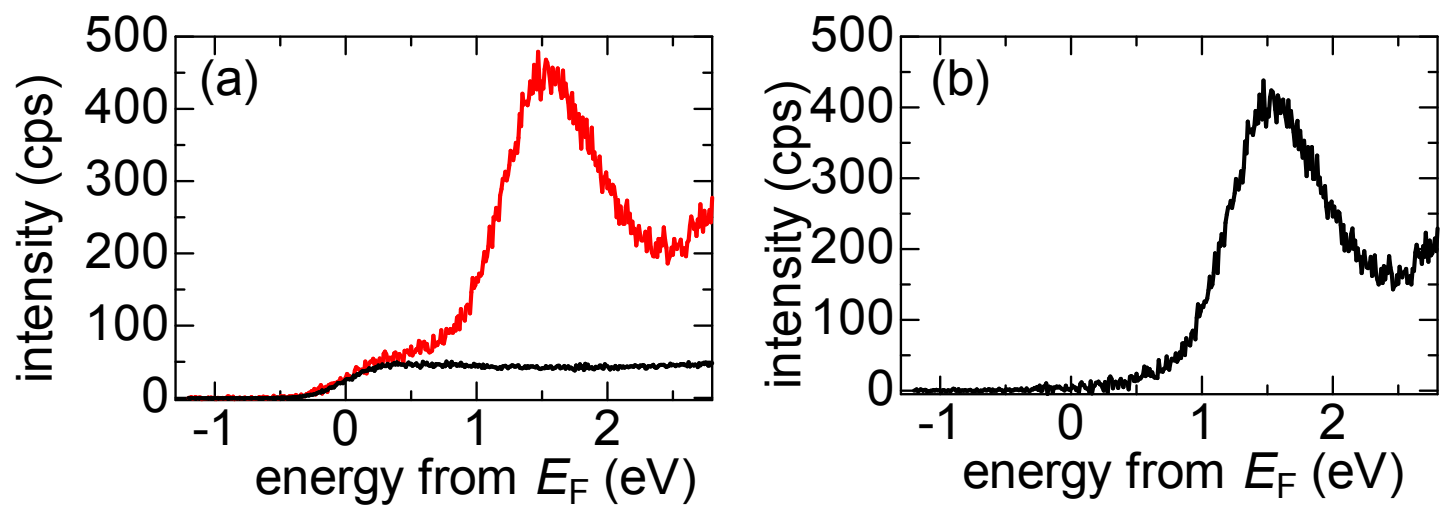

Figure S4. (a) LEIPS spectra of CuPc $(5 \mathrm{~nm}) / \mathrm{Ag}(3 \mathrm{~nm})$ and $\mathrm{Ag}(3 \mathrm{~nm})$ multiplied by 0.15 shown by the red and black lines, respectively. (b) Difference spectrum corresponding to CuPc-derived LEIPS spectrum. 